\title{
Interference Alignment Inspired Opportunistic Communications in Multi-Cluster MIMO Networks with Wireless Power Transfer
}

\author{
Yuan $\operatorname{Ren}^{1}{ }^{1}$, Xuewei Zhang ${ }^{1, *}$ and Meruyert Makhanbet ${ }^{2}$ \\ 1 Shaanxi Key Laboratory of Information Communication Network and Security, Xi'an University of Posts and \\ Telecommunications, Xi'an 710121, China; renyuan@xupt.edu.cn \\ 2 School of Information and Communication Engineering, Beijing University of Posts and Telecommunications, \\ Beijing 100876, China; mika.makhanbet@bupt.edu.cn \\ * Correspondence: zhangxw@xupt.edu.cn
}

Citation: Ren, Y.; Zhang, X.; Makhanbet, M. Interference Alignment Inspired Opportunistic Communications in Multi-Cluster MIMO Networks with Wireless Power Transfer. Information 2021, 12, 335. https://doi.org/10.3390/ info12080335

Academic Editor: Corinna Schmitt

Received: 17 July 2021

Accepted: 20 August 2021

Published: 21 August 2021

Publisher's Note: MDPI stays neutral with regard to jurisdictional claims in published maps and institutional affiliations.

Copyright: (c) 2021 by the authors. Licensee MDPI, Basel, Switzerland. This article is an open access article distributed under the terms and conditions of the Creative Commons Attribution (CC BY) license (https:/ / creativecommons.org/licenses/by/ $4.0 /)$.

\begin{abstract}
In this work, we jointly investigate the issues of node scheduling and transceiver design in a sensor network with multiple clusters, which is endowed with simultaneous wireless information and power transfer. In each cluster of the observed network, $S$ out of $N$ nodes are picked, each of which is capable of performing information transmission (IT) via uplink communications. As for the remaining idle nodes, they can harvest energy from radio-frequency signals around their ambient wireless environments. Aiming to boost the intra-cluster performance, we advocate an interference alignment enabled opportunistic communication (IAOC) scheme. This scheme can yield better tradeoffs between IT and wireless power transfer (WPT). With the aid of IAOC scheme, the signal projected onto the direction of the receive combining vector is adopted as the accurate measurement of effective signal strength, and then the high-efficiency scheduling metric for each node can be accordingly obtained. Additionally, an algorithm, based on alternative optimization and dedicated for transceiver design, is also put forward, which is able to promote the achievable sum rate performance as well as the total harvested power. Our simulation results verify the effectiveness of the designed IAOC scheme in terms of improving the performance of IT and WPT in multi-cluster scenarios.
\end{abstract}

Keywords: interference alignment; node scheduling; opportunistic communications; wireless power transfer; alternative optimization; Internet of Things

\section{Introduction}

Currently, we have witnessed the fast soaring of the number of Internet of Things (IoT) equipment [1]. Owing to the rapid development and widespread deployment of IoT, $11 \%$ economical gains are predicted to be brought at the end of 2025 [2]. With plenty of IoT equipment and diverse applications surrounding us, our world has indeed become a global village, where we can readily get access to unprecedented convenience for living, working and studying. Typically, the IoT network is complicated, since there are numerous fundamental network components to support diverse practical IoT applications. Among many network components of IoT architecture, the multi-cluster sensor network is often regarded as one of the basic paradigms. Under the presence of severe co-channel interference (CCI) and scarce bandwidth resource, it is a tough task to enhance the spectrum efficiency (SE) for the multi-cluster sensor networks.

To effectively deal with the severe CCI appearing in multi-cluster sensor networks, interference alignment (IA) can be adopted as a powerful technique for interference management [3,4]. Till now, IA has shown great potential in multiple-input multiple-output (MIMO) scenario, and can acquire the optimal degree-of-freedom (DoF) performance of interference networks in the ultra high signal-to-noise (SNR) ratio cases [5]. IA has been widely studied in cellular network [6,7], IoT network [8,9], heterogeneous network (HetNet) $[10,11]$, cache-enable network [12] and small cell network [13] to make the wireless networks perform in a spectrum-efficient manner. In [10], an IA-based set optimization 
method is proposed to solve the interference management problem of HetNets, which can achieve full diversity with limited information exchange.

In this paper, an IA enabled opportunistic communication (IAOC) scheme is proposed for SWIPT-enabled sensor networks. To be more specific, $S$ multi-antenna nodes $(S \geq 2)$ are picked out of $N$ candidates $(N \geq S)$ for each cluster, and we concentrate on the cochannel uplink transmission. When performing the uplink transmission process, the $(N-S)$ nodes that are not selected are able to realize the function of energy harvesting, and the harvested energy comes from the $S$ selected nodes belonging to each cluster. In particular, a joint node scheduling and transceiver alternating optimization algorithm is put forward, aiming for obtaining the optimal achievable sum rate and harvested energy with partial CSI at each node. The scheduling metric for IAOC is delicately devised to capture the capabilities of IT and EH. The IT capability refers to ratio between the effective signal strength of each node and the generated interference, and the EH capability is measured by the amount of energy that a node can provide. Aiming to provide a practical and unified performance metric to describe both IT and EH capabilities, the effective signal strength of each node is represented by the signal strength that is projected to the receive combining vector belonging to the cluster head $(\mathrm{CH})$. Moreover, the transmit beamforming vector and receive combining vector are iteratively optimized during each transmission block by the proposed alternating optimization algorithm. In Comparison to the former OIA schemes [14,15], the proposed IAOC framework is able to balance IT and EH by adjusting the weight factor in the considered SWIPT-enabled sensor network.

The rest of this paper is organized as follows. In Section 2, we specify the system model. Section 3 formulates the joint IT and EH optimization problem, and the alternating optimization based IAOC scheme is also presented. Numerical results are provided and analyzed in Section 4. Section 5 gives the concluding remarks of this paper.

Notations: In our work, we use the lowercase boldface letters for vectors and use the uppercase boldface letters for matrices. $\|\cdot\|$ and $(\cdot)^{H}$ represent the Euclidean-norm and conjugate transpose, respectively. $[1, K]$ represents the integer set $\{1,2, \ldots, K\} . \mathbf{I}_{m}$ denotes the $m \times m$ identity matrix. $\kappa_{n}(\mathbf{A}, \mathbf{B})$ refers to the $n$-th largest generalized eigenvalue of $\mathbf{A}$ and $\mathbf{B}$, and $\mathbf{w}_{n}(\mathbf{A}, \mathbf{B})$ refers to the associating generalized eigenvector. $\left(\begin{array}{l}n \\ k\end{array}\right)$ represents the number of $k$-combinations from a given set of $n$ elements.

\section{Related Work}

Although IA owns theoretical advantages, there still exist many challenges for practical applications of IA. In particular, the global channel state information (CSI) at each transceiver is often demanded for the conventional IA schemes [16-18]. In addition, owing to the expansions in terms of time, frequency or symbol, perfect IA transceiver design requires a large number of signal dimensions $[3,5,6]$. In order to reduce the CSI overhead in the system, IA strategies leveraging limited feedback are advocated in $[19,20]$. However, for the IA schemes based on limited feedback, the length of the feedback signal should scale with the transmitted power to achieve DoF gain. In [21], the authors investigate a $K$-user $M \times N$ MIMO interference channel with delayed channel state information at the transmitters, and propose a novel method that applies the IA procedure at the receiver side. More recently, a robust joint interference and phase alignment scheme based on Bayesian estimation and power allocation is proposed in [22] to overcome time delay error CSI in the MIMO broadcasting communication system.

To let the technique of IA make more practical sense, opportunistic interference alignment (OIA) has been developed for the networks with multiple cells or clusters in both downlink [23-26] and uplink $[14,15,27,28]$ transmissions. In this regard, OIA has many advantages over the traditional IA schemes $[29,30]$. To be more specific, OIA can be implemented with local CSI and the expansion of symbol, time or frequency is not required. For the downlink OIA, the chordal distance is used as the scheduling metric to characterize the degree of alignment, and only one user, whose interference signal is the most aligned for information transmission (IT), is chosen by each base station (BS) [23]. Considering the 
leakage arising from interference and desired signals when devising the OIA scheduling metric, the performance of downlink sum rate can be further boosted by [24]. In [25], a joint transmit/receive beamforming and user scheduling scheme is designed, which fundamentally reduces the user scaling law as shown in [23], and a limited feedback strategy is analyzed. Additionally, for the OIA strategy, the performance of achievable sum rate is analyzed in MIMO interfering broadcast channels in [26].

In the realization of the uplink OIA, a set of vectors that are orthonormal and random with each other is termed as the reference signal subspace (RSS). By broadcasting RSS to all users, the direction of data transmission can be guided [14,27,28]. In [27,28], an OIA scheme is studied for single-input multiple-output (SIMO) interfering multiple-access channel (IMAC), which can make the interference signal align to the well-designed interference spaces for each BS. An OIA-assisted singular value decomposition (SVD) is advocated for the MIMO IMAC scenario [14], and both transmit beamforming and user scheduling are applied for the proposed scheme. In a word, the essence of $[14,27,28]$ is to pick the users whose generated interference has the minimum effect on other cells. Although the scheme of minimizing leakage interference (Min-LIF) is DoF optimal in very high SNR regimes, the sum rate improvement is supposed to be considered in more practical SNR cases. Aiming for further boosting the sum rate performance, a threshold inspired scheme is presented in [15], where the balance between the strength of desired signal and leakage interference is included for consideration.

In recent years, simultaneous wireless information and power transfer (SWIPT) turns into one of the most important applications in the field of energy harvesting (EH) [31,32]. Apart from eliminate interference in multi-cluster sensor networks, by properly designing the transmission scheme, the various types of CCI can also be effectively utilized to enable efficient power transfer. Coupling IA with SWIPT, some primary work has been conducted in [33-37]. Specifically, in [35], the antenna selection is investigated to obtain the optimal sum rate and total harvested energy. In [36], the optimal angle switching is studied for the SWIPT-enabled IA network. However, these studies only consider the case where we can handily obtain the global CSI [33-36] and perfect IA can be implemented. If perfect IA cannot be performed, this will restrain them from being extended to more general scenarios $[14,15]$. In addition, an effective scheme for imperfect IA and EH design is proposed in [37], where the pseudo DoFs that are not available in practical iterative IA process is exploited for EH. Unfortunately, the node scheduling is not included in [37]. As far as we know, there is little known about the joint study of node scheduling and transceiver design without global CSI for the general SWIPT-enabled multi-cluster sensor networks.

In summary, this paper jointly studies node scheduling and transceiver design in SWIPT-enabled multi-cluster MIMO networks, and the IAOC scheme is proposed to facilitate efficient information and power transfer.

\section{System Model}

As shown in Figure 1, an SWIPT-enabled K-cluster sensor network is observed. In particular, each cluster is comprised by one $\mathrm{CH}$ and $\mathrm{N}$ nodes. It is assumed that the $\mathrm{CH}$ has $N_{r}$-antenna and each node is deployed with $N_{t}$-antenna $\left(N \geq N_{r}\right)$. The set of nodes for the $k$-th cluster is represented by $\Pi_{k}=\left\{\mathrm{F}_{k, 1}, \mathrm{~F}_{k, 2}, \ldots, \mathrm{F}_{k, N}\right\}, k \in[1, K]$. In each cluster, $S$ nodes will be selected to transmit information to its corresponding $\mathrm{CH}$ and conduct wireless power transfer to the remaining $(N-S)$ unselected nodes. The set of selected nodes in the $k$-th cluster is denoted by $\Xi_{k}=\left\{\xi_{k}(1), \ldots, \xi_{k}(S)\right\}$, and the set for unselected nodes is represented by $\Lambda_{k}=\left\{\lambda_{k}(1), \ldots, \lambda_{k}(N-S)\right\}, k \in[1, K]$. 


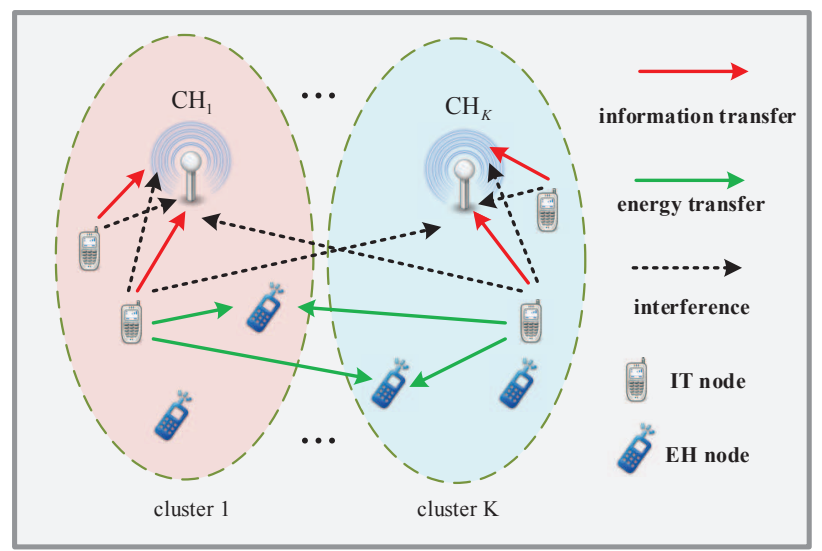

Figure 1. System model for K-cluster SWIPT-enabled sensor network. In each cluster, $S$ nodes are selected from $N$ nodes to send data streams to its corresponding $\mathrm{CH}$, and the remaining $(N-S)$ nodes can conduct wireless $\mathrm{EH}$ to recharge their batteries.

After node scheduling, the received signal $\mathbf{y}_{k}$ at $\mathrm{CH}_{k}$, whose size is $\mathrm{N}_{r} \times 1$, is written as

$$
\mathbf{y}_{k}=\sum_{j \in \Xi_{k}} \theta_{k, j}^{[k]} \mathbf{G}_{k, j}^{[k]} \mathbf{v}_{k, j} x_{k, j}+\sum_{i=1, i \neq k}^{K} \sum_{j \in \Xi_{i}} \theta_{i, j}^{[k]} \mathbf{G}_{i, j}^{[k]} \mathbf{v}_{i, j} x_{i, j}+\mathbf{n}_{k}
$$

where $x_{i, j}$ denotes the information symbol transmitted from $\mathrm{F}_{i, j}$ to $\mathrm{CH}_{i}, i \in[1, K], j \in \Xi_{i}$; $\mathbf{v}_{i, j}$ represents the well-designed transmit beamforming vector of $\mathrm{F}_{i, j}, i \in[1, K], j \in \Xi_{i}$; $\mathbf{n}_{k} \in \mathbb{C}^{N_{r} \times 1}$ donotes the complex additive antenna noise vector at $\mathrm{CH}_{k}$, following $\mathbf{n}_{k} \sim$ $\mathcal{C N}\left(0, \sigma^{2} \mathbf{I}_{N_{r}}\right) ; P_{t}$ is the maximum transmission power at $\mathrm{F}_{i, j}$, i.e., $\mathrm{E}\left[\left|x_{k, j}\right|^{2}\right] \leq P_{t}$. We assume the uncorrelated antenna channels, and this assumption is consistent with the existing works on OIA $[14,15,23-28] . \mathbf{G}_{i, j}^{[k]}$ denotes the channel matrix caused by smallscale fading from $\mathrm{F}_{i, j}$ to $\mathrm{CH}_{k}, i, k \in[1, K], j \in \Xi_{i}$; and $\theta_{i, j}^{[k]}$ represents the corresponding path-loss coefficient from $\mathrm{F}_{i, j}$ to $\mathrm{CH}_{k}$. We suppose that each element appearing in $\mathrm{G}_{i, j}^{[k]}$ is independent and identically distributed (i.i.d.), according to the distribution $\mathcal{C N}\left(0, \delta^{2}\right)$. It is also assumed that only partial CSI is known at each node. That is to say, $\mathrm{CH}_{k}$ knows the CSI from the $N$ nodes within the cluster $\Pi_{k}$, and $\mathrm{F}_{i, j}$ possesses the CSI from the total $\mathrm{KCHs}$ and $(N-1)$ nodes within the cluster $\Pi_{k}-\left\{\mathrm{F}_{i, j}\right\}$. The instantaneous signal-to-interferenceplus-noise ratio (SINR) of $\mathrm{F}_{k, j}$ is given by

$$
\operatorname{SINR}_{k, j}=\frac{\rho\left|\theta_{k, j}^{[k]} \mathbf{u}_{s}^{[k] H} \mathbf{G}_{k, j}^{[k]} \mathbf{v}_{k, j}\right|^{2}}{\rho\left(\sum_{i=1}^{K} \sum_{j \in \Xi_{i}}\left|\theta_{i, j}^{[k]} \mathbf{u}_{s}^{[k] H} \mathbf{G}_{i, j}^{[k]} \mathbf{v}_{i, j}\right|^{2}-\left|\theta_{k, j}^{[k]} \mathbf{u}_{s}^{[k] H} \mathbf{G}_{k, j}^{[k]} \mathbf{v}_{k, j}\right|^{2}\right)+1},
$$

where $\mathbf{U}^{[k]}=\left[\mathbf{u}_{1}^{[k]}, \ldots, \mathbf{u}_{S}^{[k]}\right] \in \mathbb{C}^{N_{r} \times S}$ is the receive combining matrix of $\mathrm{CH}_{k}$, and $\rho$ is the transmit SNR. Therefore, the sum rate of the considered multi-cluster scenario can be accordingly written as

$$
\mathrm{R}_{\mathrm{sum}}=\sum_{k=1}^{K} \sum_{j \in \Xi_{k}} \log \left(1+\operatorname{SINR}_{k, j}\right) .
$$

when the $S$ nodes located in each cluster are selected for IT, the $(N-S)$ unselected nodes remain idle, and can harvest wireless energy to recharge their batteries from the selected 
nodes. In this regard, the received signal at the idle nodes without data transmission, e.g., $\left\{\mathrm{F}_{k, s}\right\}, s \in \Lambda_{k}$, can be represented as

$$
\mathbf{y}_{k, s}=\sum_{i=1}^{K} \sum_{j \in \Xi_{i}} \theta_{i, j}^{[k, s]} \mathbf{G}_{i, j}^{[k, s]} \mathbf{v}_{i, j} x_{i, j}+\mathbf{n}_{k, s}
$$

where $\mathbf{n}_{k, s} \in \mathbb{C}^{N_{t} \times 1}$ denotes the circuit noise vector at $\mathrm{F}_{k, s}$, satisfying $\mathbf{n}_{k, s} \sim \mathcal{C N}\left(0, \sigma^{2} \mathbf{I}_{N_{t}}\right)$; $\theta_{i, j}^{[k, s]}$ is the path-loss; and $\mathbf{G}_{i, j}^{[k, s]}$ is the small-scale fading. We make an assumption that each element of $\mathbf{G}_{i, j}^{[k, s]}$ is i.i.d., following $\mathcal{C N}\left(0, \delta^{2}\right)$. Then, the power that is expected to be harvested can be reserved at $\mathrm{F}_{k, s}$. This kind of energy is denoted by $\mathrm{Q}_{k, s}$, and can be expressed as

$$
\mathrm{Q}_{k, s}=\eta \sum_{i=1}^{K} \sum_{j \in \Xi_{i}}\left\|\theta_{i, j}^{[k, s]} \mathbf{G}_{i, j}^{[k, s]} \mathbf{v}_{i, j} x_{i, j}\right\|^{2},
$$

where $0<\eta<1$ denotes the energy conversion efficiency from radio-frequency (RF) signals to electric energy [36]. Among the expected harvested power, the power strength of the noise is much smaller than that of the average received signal, and the energy harvested from the noise power contributes little to the total harvested power. As a consequence, this part of harvested energy is overlooked [31]. To this end, the total harvested power in the SWIPT-enabled sensor network can be calculated by

$$
\mathrm{Q}_{\mathrm{sum}}=\sum_{k=1}^{K} \sum_{s \in \Lambda_{k}} \mathrm{Q}_{k, s} .
$$

\section{The Proposed IAOC Scheme}

In this section, the joint node scheduling and transceiver design is carried out to improve the performance of IT and EH. Inspired by the idea of SWIPT, interference, as a special form of ambient RF signal, bears the potential to enable more efficient usage and wireless power transfer. For the previous OIA schemes, the design of scheduling metrics focuses on characterizing the signal strength of leakage interference or the demanded signal, and the objective of these schemes is to enhance the performance of achievable sum rate. In light of the above observation, in the SWIPT-enabled sensor network, we formulate the joint optimization problem (JOP) as

$$
\max _{\mathbf{v}_{k, j}, \mathbf{U}^{[k]}, \Xi_{k}} \sum_{k=1}^{K} \sum_{j \in \Xi_{k}} \log \left(1+\operatorname{SINR}_{k, j}\right)+\beta \sum_{k=1}^{K} \sum_{s \in \Lambda_{k}} \mathrm{Q}_{k, s},
$$

where $\beta$ is a constant parameter in $\mathrm{bps} / \mathrm{Hz} / \mathrm{W}$, used to trade off the importance between the rate and power [34]. The JOP is a combinatorial optimization problem, which is proved to be non-convex. The centralized algorithm for solving this problem will result in high computational complexity. To this end, a algorithm for node scheduling and transceiver alternating optimization is proposed to simultaneously optimize the achievable sum rate and the harvested power.

\subsection{Scheduling Metric Design}

For the considered SWIPT-enabled sensor network, $S$ scheduling metrics are defined for each node to characterize the IT and WPT performance, and each scheduling metric is associated with a certain receive combining vector. To be more specific, the $s$-th scheduling metric of $\mathrm{F}_{k, j}$, corresponding to the receive combining vector $\mathbf{u}_{s}$, can be represented as 


$$
\begin{aligned}
\mathbf{M}_{k, j}(s) & =\frac{\alpha_{k, j}\left|\theta_{k, j}^{[k]} \mathbf{u}_{s}^{[k] H} \mathbf{G}_{k, j}^{[k]} \mathbf{v}_{k, j}\right|^{2}+\left(1-\alpha_{k, j}\right) \eta \sum_{i=1, i \neq j}^{N}\left\|\theta_{k, j}^{[k, i]} \mathbf{G}_{k, j}^{[k, i]} \mathbf{v}_{k, j}\right\|^{2}}{\sum_{i=1, i \neq k}^{K}\left\|\theta_{k, j}^{[i]} \mathbf{U}^{[i]} \mathbf{G}_{k, j}^{[i]} \mathbf{v}_{k, j}\right\|^{2}} \\
& =\frac{\mathbf{v}_{k, j}^{H} \mathbf{C}_{k, j}(s) \mathbf{v}_{k, j}}{\mathbf{v}_{k, j}^{H} \mathbf{D}_{k, j} \mathbf{v}_{k, j}},
\end{aligned}
$$

where $\mathbf{C}_{k, j}(s)$ and $\mathbf{D}_{k, j}$ can be expressed as

$$
\begin{aligned}
& \mathbf{C}_{k, j}(s)=\alpha_{k, j}\left|\theta_{k, j}^{[k]}\right|^{2} \mathbf{G}_{k, j}^{[k] H} \mathbf{u}_{s}^{[k]} \mathbf{u}_{s}^{[k] H} \mathbf{G}_{k, j}^{[k]}+\eta\left(1-\alpha_{k, j}\right) \sum_{i=1, i \neq j}^{N}\left|\theta_{k, j}^{[k, i]}\right|^{2} \mathbf{G}_{k, j}^{[k, i] H} \mathbf{G}_{k, j}^{[k, i]}, \\
& \mathbf{D}_{k, j}=\sum_{i=1, i \neq k}^{K}\left|\theta_{k, j}^{[i]}\right|^{2} \mathbf{G}_{k, j}^{[i] H} \mathbf{U}^{[i]} \mathbf{U}^{[i] H} \mathbf{G}_{k, j}^{[i]}
\end{aligned}
$$

and $\alpha_{k, j}\left(0 \leq \alpha_{k, j} \leq 1\right)$ is the weight factor. When $\alpha_{k, j}$ is large, it indicates the IT requirement is strict or the battery power is adequate. On the contrary, a small value of $\alpha_{k, j}$ represents that the IT requirement is low or the battery power is not adequate. As exhibited in (8), the denominator of $\mathrm{M}_{k, j}(s)$ yields the leakage interference from $\mathrm{F}_{k, j}$ leaked to the other $(K-1)$ clusters. The first term of the numerator for $\mathrm{M}_{k, j}(s)$ quantifies the effective signal strength projected on each dimension of the receive combining matrix as a substantially refined description of the intra-cluster transmission performance. The second term of the numerator for $\mathrm{M}_{k, j}(s)$ calculates the possible harvested power in the cluster provided by $\mathrm{F}_{k, j}$. Based on the scheduling metric defined above, the performance of IT and EH are both considered for node scheduling. Moreover, from the perspective of IT, the effective signal strength of the node, i.e., the projection in the direction of each receive combining vector, and the leakage interference are optimized to boost the achievable sum rate in the considered sensor network. On the basis of the Rayleigh-Ritz theorem [38], we can calculate the optimal scheduling metric $\mathrm{M}_{k, j}(s)$ by employing the approach of generalized eigenvalue decomposition [39,40], and the optimal value of $\mathrm{M}_{k, j}(s)$ is given as follows:

$$
\mathbf{M}_{k, j}^{*}(s)=\kappa_{1}\left(\mathbf{C}_{k, j}(s), \mathbf{D}_{k, j}\right),
$$

where $\kappa_{1}\left(\mathbf{C}_{k, j}(s), \mathbf{D}_{k, j}\right)$ is the largest generalized eigenvalue of $\mathbf{C}_{k, j}(s)$ and $\mathbf{D}_{k, j}$.

\subsection{Node Scheduling}

According to the feedback scheduling metrics of the nodes, $\mathrm{CH}_{k}$ is able to conduct the node selection. $S$ nodes will be selected by $\mathrm{CH}_{k}$ corresponding to the $S$ receive combining vectors of $\mathrm{CH}_{k}$. In particular, for a certain receive combining vector $\mathbf{u}_{s}^{[k]}$ of $\mathrm{CH}_{k}$, the node with the largest value of $\mathrm{M}_{k, j}^{*}(s)$ will be selected by $\mathrm{CH}_{k}$. Therefore, $\mathrm{CH}_{k}$ selects the node according to the following principle:

$$
\xi_{k}(s)=\underset{j \in \Lambda_{k}}{\arg \max } \mathbf{M}_{k, j}^{*}(s), k \in[1, K], s \in[1, S] .
$$

\subsection{Transmit Beamforming Vector Design}

When the process of node selection is finished, the scheduling information will be broadcast by $\mathrm{CH}_{k}$, and the selected node $\mathrm{F}_{k, \xi_{k}(s)}$ is capable of obtaining the corresponding 
reference direction $\mathbf{u}_{s}^{[k]}$ as well. Afterwards, according to (9), the selected node $\mathrm{F}_{k, \tilde{\zeta}_{k}(s)}$ calculates the transmit beamforming vector as

$$
\mathbf{v}_{k, \xi_{k}(s)}^{*}=\underset{\mathbf{v}_{k, j}}{\arg \max } \frac{\mathbf{v}_{k, j}^{H} \mathbf{C}_{k, j}^{*}(s) \mathbf{v}_{k, j}}{\mathbf{v}_{k, j}^{H} \mathbf{D}_{k, j} \mathbf{v}_{k, j}},
$$

where $\mathbf{C}_{k, j}^{*}(s)$ can be expressed as

$$
\mathbf{C}_{k, j}^{*}(s)=\alpha_{k, j}\left|\theta_{k, j}^{[k]}\right|^{2} \mathbf{G}_{k, j}^{[k] H} \mathbf{u}_{s}^{[k]} \mathbf{u}_{s}^{[k] H} \mathbf{G}_{k, j}^{[k]}+\eta\left(1-\alpha_{k, j}\right) \sum_{i \in \Lambda_{k}}\left|\theta_{k, j}^{[k, i]}\right|^{2} \mathbf{G}_{k, j}^{[k, i] H} \mathbf{G}_{k, j}^{[k, i]} .
$$

Therefore, according to the Rayleigh-Ritz theorem [38], when the selected node set and receive combining vector are given, the optimal transmit beamforming vector design can be yielded by maximizing the value of the scheduling metric in (9). Applying the approach of generalized eigenvalue decomposition $[39,40]$, the optimal transmit beamforming vector of $\mathrm{F}_{k, \xi_{k}(s)}$ can be expressed as

$$
\mathbf{v}_{k, \xi_{k}(s)}^{*}=\mathbf{w}_{1}\left(\mathbf{C}_{k, j}^{*}(s), \mathbf{D}_{k, j}\right),
$$

where $\mathbf{w}_{1}\left(\mathbf{C}_{k, j}^{*}(s), \mathbf{D}_{k, j}\right)$ is the generalized eigenvector associating with the largest generalized eigenvalue $\kappa_{1}\left(\mathbf{C}_{k, j}(s), \mathbf{D}_{k, j}\right)$.

In the devised scheduling metric shown in (12) as well as the transmit beamforming design presented in (16), only the harvested power arising from intra cluster is included. This is different from (5) where the harvested power sourced from both intra and inter clusters is taken into account. The reasons for this design can be illustrated as follows. First of all, owing to the co-existing path loss, the harvested power of inter cluster will be smaller than that of intra cluster for each unselected node. Moreover, due to the fact that the increasing speed of the overheads spent on CSI acquisition will be highly fast when the number of nodes is very large, it is not a wise choice for each node to possess all CSI information related to the nodes located in other clusters.

\subsection{Receive Combining Vector Design}

When the transmit beamforming vectors are obtained, the selected nodes can begin the uplink transmission process. The receive combining vector is optimized to maximized $\mathrm{SINR}_{k, j}$ in (2) at $\mathrm{CH}_{k}$ as follows:

$$
\mathbf{u}_{s}^{[k] *}=\underset{\mathbf{u}_{s}^{[k]}}{\arg \max } \frac{\mathbf{u}_{s}^{[k] H} \mathbf{X}_{k, j} \mathbf{u}_{s}^{[k]}}{\mathbf{u}_{s}^{[k] H} \mathbf{Y}_{k, j} \mathbf{u}_{s}^{[k]}},
$$

where we have

$$
\begin{aligned}
& \mathbf{X}_{k, j}=\rho\left|\theta_{k, j}^{k}\right|^{2} \mathbf{G}_{k, j}^{[k]} \mathbf{v}_{k, j} \mathbf{v}_{k, j}^{H} \mathbf{G}_{k, j}^{[k] H}, \\
& \mathbf{Y}_{k, j}=\rho\left(\sum_{i=1}^{K} \sum_{j \in \Xi_{i}}\left|\theta_{i, j}^{k}\right|^{2} \mathbf{G}_{i, j}^{[k]} \mathbf{v}_{i, j} \mathbf{v}_{i, j}^{H} \mathbf{G}_{i, j}^{[k] H}-\left|\theta_{k, j}^{k}\right|^{2} \mathbf{G}_{k, j}^{[k]} \mathbf{v}_{k, j} \mathbf{v}_{k, j}^{H} \mathbf{G}_{k, j}^{[k] H}\right)+\mathbf{I}_{N_{r}} .
\end{aligned}
$$

Similar to the method described in Section 4.3, with the aid of the Rayleigh-Ritz theorem [38], we can obtain the optimal receive combining vector of $\mathbf{u}_{s}^{[k]}$ by adopting the approach of generalized eigenvalue decomposition [39,40], which is given as

$$
\mathbf{u}_{s}^{[k] *}=\mathbf{w}_{1}\left(\mathbf{X}_{k, j}, \mathbf{Y}_{k, j}\right)
$$

and the corresponding $\operatorname{SINR}_{k, j}=\kappa_{1}\left(\mathbf{X}_{k, j}, \mathbf{Y}_{k, j}\right)$. 
During the first transmission block, the receive combining matrix $\mathbf{U}^{[k]}$ is initialized by the RSS of $\mathrm{CH}_{k}$, i.e., $\mathbf{W}^{[k]}=\left[\mathbf{w}_{1}^{[k]}, \ldots, \mathbf{w}_{S}^{[k]}\right] \in \mathbb{C}^{N_{r} \times S}$. This matrix is semi-unitary, and satisfies $\mathbf{W}^{[k] H} \mathbf{W}^{[k]}=\mathbf{I}_{S}$. The alternating optimization algorithm for IAOC is summarized as Algorithm 1.

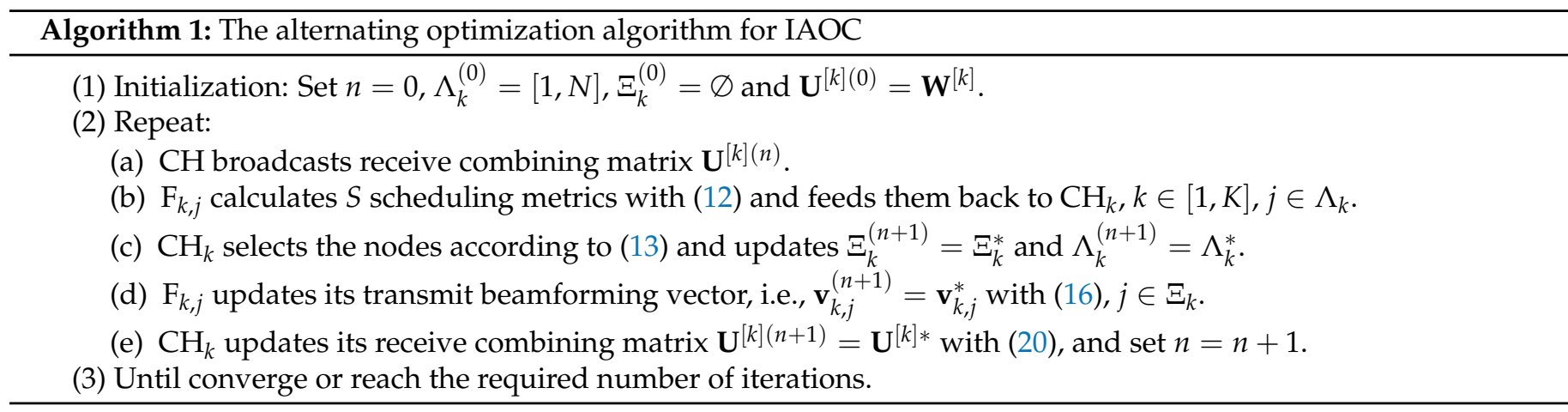

Remark 1. As mentioned in Section $4.1, M_{k, j}(s)$ represents the s-th scheduling metric of $F_{k, j}$ corresponding to the receive combining vector $\mathbf{u}_{s}$. The denominator of $M_{k, j}(s)$ represents the leakage interference, i.e., the inter-cluster interference leaked from $F_{k, j}$ to the other $(K-1)$ clusters. The node with less leakage interference has a larger scheduling metric and is more likely to be selected. The first term of the numerator of $M_{k, j}(s)$ characterizes the intra-cluster performance, which quantifies the effective signal strength projected on the receive combining vector $\mathbf{u}_{s}$. The $S$ receive combining vectors can constitute a reference signal space, and can be viewed as a group of reference directions to guide node selection in the cluster. For each receive combining vector, the node that is more aligned to the direction will have a larger scheduling metric and is more likely to be selected. Similarly, the design of the transmit beamforming and receive combining vector also considers both intra-cluster and inter-cluster interference. To sum up, the intra-cluster and inter-cluster interference are both considered in the node scheduling and transceiver design of the proposed scheme.

\subsection{Feedback Overhead and Computational Complexity}

Firstly, we use $\epsilon_{1} \in(0,1)$ to denote the probability that the node meeting the two conditions (C1) and (C2) [15]. Then, the feedback overheads of the Min-LIF scheme, the threshold based OIA and the IAOC are one, $2 S \varepsilon_{1}$ and $S$ scalar values, respectively.

The computational complexity of each strategy is quantitatively evaluated via the number of floating-point operations (FLOPs). Specifically, multiplication or division of real numbers consists of one flop; each addition operation of complex numbers costs two-flop complexity; and each multiplication operation of complex numbers includes six flops. Bearing the aforementioned principle in mind, the computational complexity cost by computing the scheduling metric and the beamforming vector can be obtained. Big $O$ function is introduced to capture the computational complexity of the devised scheme, which can cover the complexity arising from matrix multiplication, eigenvalue decomposition and other mathematical operations. With the assistance of big $O$ function, in Min-LIF based and threshold based strategies, the computational complexities per node are calculated as $O\left(K S N_{r} N_{t}+K S N_{t}^{2}\right)$ [14] and $O\left(K S N_{r}^{2} N_{t}+K S^{2} N_{t}^{2}\right)$, respectively. Additionally, let $N_{I}$ denote the number of required iterations in IAOC strategy, and the per-node complexity of this scheme can be given by $O\left(N_{I} N S N_{t}^{3}+N_{I} K S N_{r} N_{t}+N_{I} K S N_{t}^{2}\right)$. Clearly, we can conclude that the computational complexity belonging to the advocated strategy is slightly higher than the complexity in the traditional OIA strategies.

\section{Numerical Simulations}

In this part, by leveraging the tool of Monte Carlo simulations, the performance of the well-designed IAOC scheme is exhibited. To accurately judge the performance gain 
brought by IAOC, the achievable sum rate and total harvested power are used to act as the criteria. As for the values of simulation parameters, it is set that $\eta=0.2, \delta=1$ and $\alpha_{k, j}=\alpha$. For simplicity but without any loss of generality, the channel model for direct/intra-cluster and cross-link/inter-cluster channels are adopted as $\theta_{k, j}^{[k]}=\theta_{k, j}^{[k, s]}=1$ and $\theta_{i, j}^{[k]}=\theta_{i, j}^{[k, s]}=0.8$, $i \neq k$, respectively, [34,36].

In Figure 2, the initial value of each entry in the receive combining vector is selected in a random manner until this vector is feasible. From this figure, we can clearly see that $R_{\text {sum }}$ of IAOC will reach its convergence within a few number of iterations. $Q_{\text {sum }}$ remains unchanged in this figure, since optimizing the receive combining vector to gain the maximum received SINR is not effective to boost the EH performance. For the purpose of performance comparison, a centralized scheme is employed as the benchmark, where the global CSI is assumed to be known in advance and the joint transceiver design is performed under each possible case of $\Xi_{k}$. In the observed network, the number of combinations of $\Xi_{k}$ is counted as $\left(\begin{array}{c}N \\ S\end{array}\right)$, and the transceiver for each $\Xi_{k}$ can arrive at its optimal performance in an iteration manner. Particularly, as the same in [41], under fixed $\mathbf{U}^{[k]}, \mathbf{v}_{k, j}$ is regarded as the optimization variable to maximize (7). Next, with updated $\mathbf{v}_{k, j}$, we can obtain the optimal $\mathbf{U}^{[k]}$ based on (20). Though the centralized strategy can be adopted as an upper bound, this scheme makes little practical sense, because the CSI overhead and the computational complexity will scale with the growing $S, N$ and $K$.

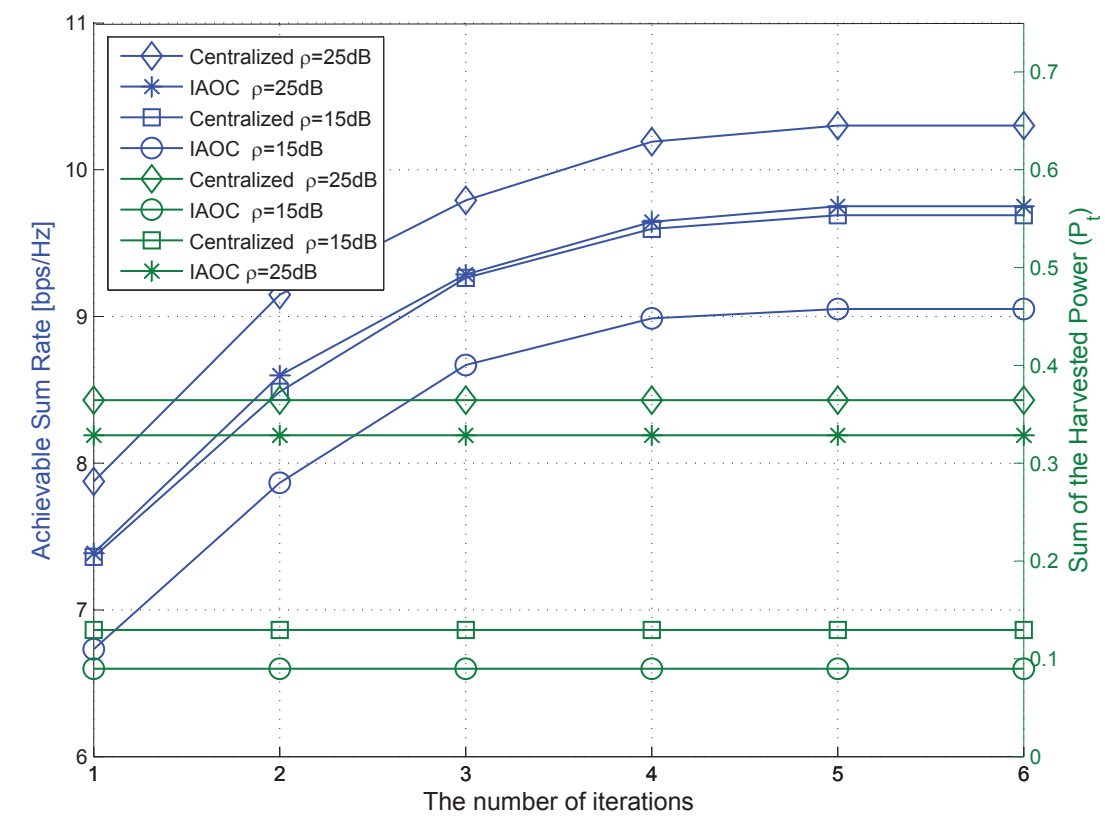

Figure 2. Convergence property of IAOC. $K=3, N_{r}=3, N_{t}=2, S=2, \alpha=0.9, N=10$.

In Figure 3, we present the performance of $R_{\text {sum }}$ under varying $N$. Under IAOC scheme, $\mathrm{R}_{\text {sum }}$ is a monotone increasing function regarding to $\alpha$. When $\alpha$ is in the range of $(0.7,1]$, IAOC yields better performance in all SNR regimes in terms of $R_{\text {sum }}$. Therefore, we can conclude that the value of $\alpha$ has a great effect on $R_{\text {sum }}$. 


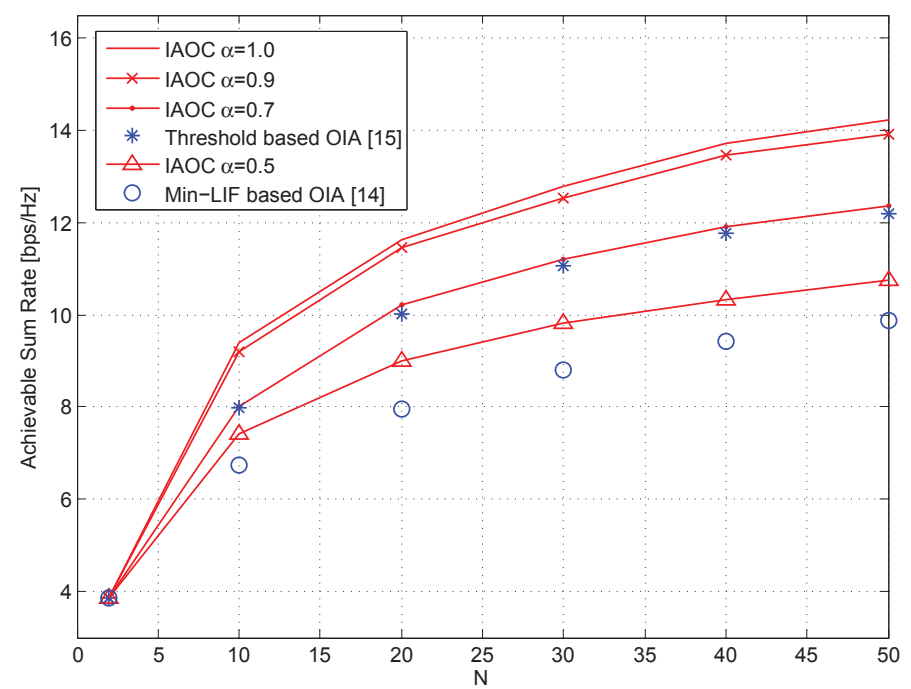

Figure 3. The achievable sum rate versus $N . K=3, N_{r}=3, N_{t}=2, S=2$.

Figure 4 shows us the relationship between $Q_{\text {sum }}$ and $N$. $Q_{\text {sum }}$ obtained from the two traditional OIA schemes are identical with each other, and these two schemes can be adopted as the benchmark strategies. From the observations of this figure, it can be found that, when $\alpha<1$, IAOC shows superior EH performance than the two benchmark schemes. As the value of $\alpha$ grows, $\mathrm{Q}_{\text {sum }}$ sourced from IAOC will decrease. From another perspective, when the value of $\alpha$ is set to 1, IAOC and two benchmarks yield the same $\mathrm{Q}_{\text {sum }}$. Based on the insights found in Figures 3 and 4, it can be concluded that $\alpha=0.9$ can make a preferable balance between achievable sum rate and total harvested power.

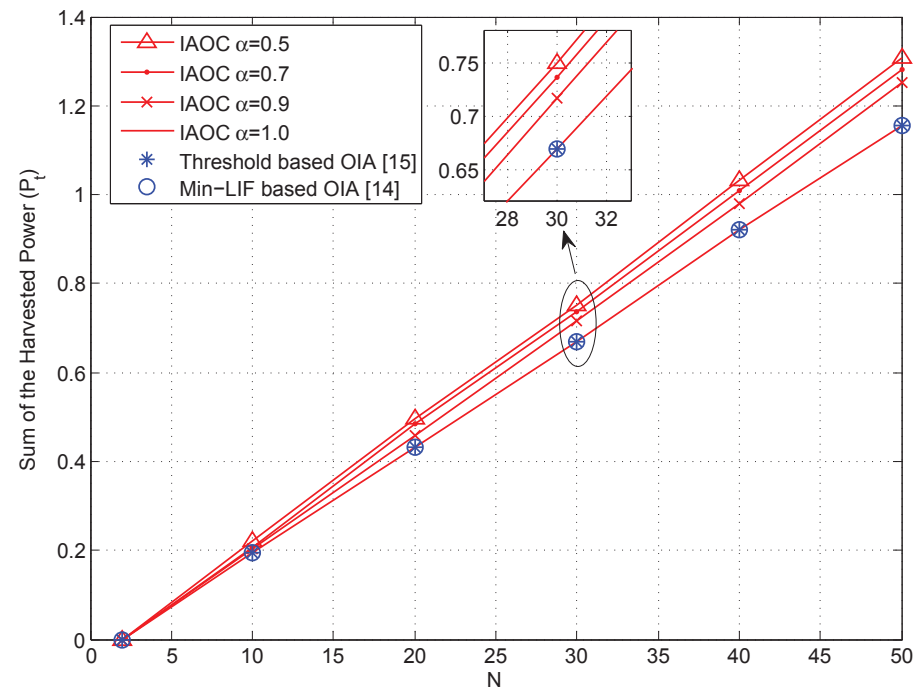

Figure 4. The total harvested power versus $N . K=3, N_{r}=3, N_{t}=2, S=2$.

Figure 5 presents the achievable sum rate for different schemes versus $N$ for $K=4$ and $N_{r}=4$. As shown in the figure, the achievable sum rate of different schemes monotonically increases with $N$. Besides, the achievable sum rate of the proposed IAOC scheme with $\alpha$ in the range of $(0.7,1]$ outperforms that of the conventional OIA schemes, which demonstrates the effectiveness of the proposed scheme. Moreover, compared with Figure 3, the achievable sum rates of all schemes decrease due to the increase of inter-cluster interference caused by the increase of $K$. 


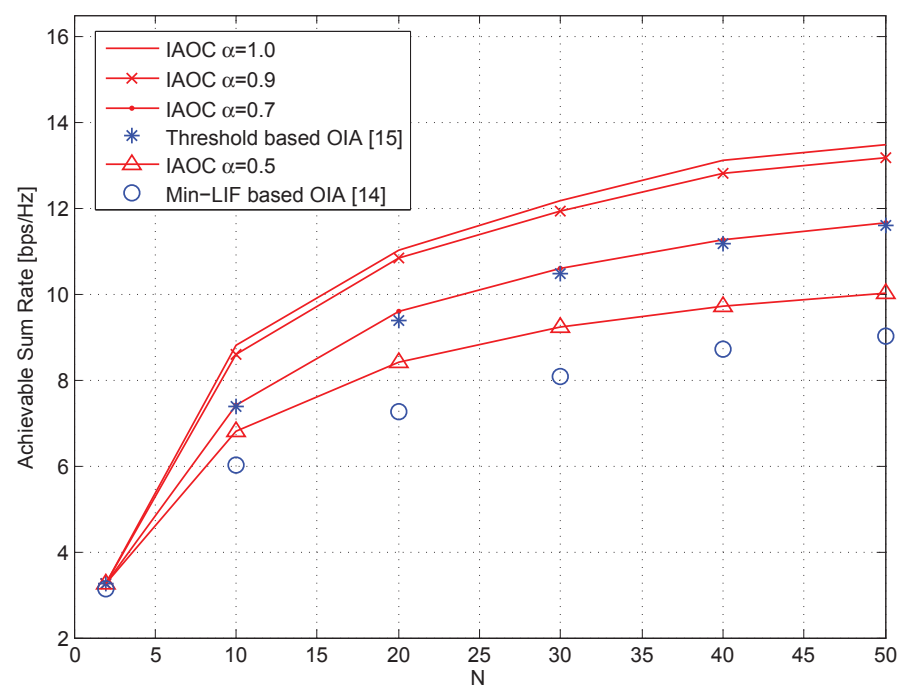

Figure 5. The achievable sum rate versus $N . K=4, N_{r}=4, N_{t}=2, S=2$.

In Figure 6, we depict the relationship between the total harvested power and $N$. It can be observed that as $N$ increases, the total harvested power is enhanced for all schemes. Moreover, compared with Figure 4, it is observed that the total harvested power of all schemes increases due to the increase of $K$. Through the analysis of Figures 5 and 6, we can find that there exists a trade-off between the IT and WPT performance of the proposed scheme. Particularly, the increase of harvested power will reduce the achievable sum rate. In this case, $\alpha=0.9$ can achieve a better trade-off between IT and WPT.

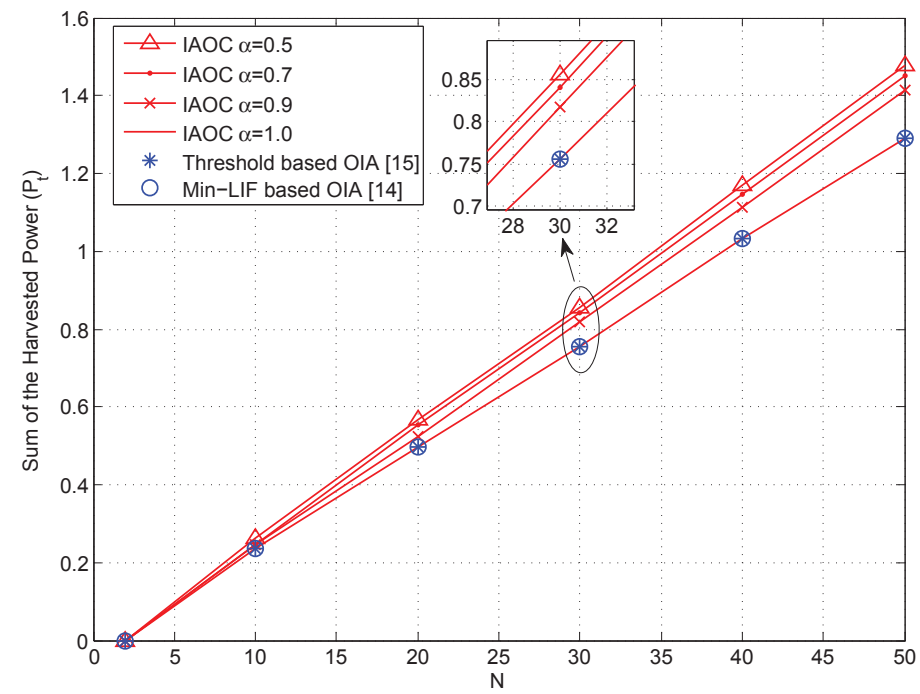

Figure 6. The total harvested power versus $N . K=4, N_{r}=4, N_{t}=2, S=2$.

\section{Conclusions}

In this work, we propose the IAOC scheme for uplink transmission in multi-cluster SWIPT-enabled sensor network. The scheduling metrics are delicately considered, and the alternating algorithm for joint optimization of achievable sum rate and total harvested power is devised. Extending the proposed IAOC scheme to more generalized scenarios considering the node's battery state is a promising future work, and the analysis for the relationship between the achievable sum rate and the harvested power in the multi-cluster SWIPT-enabled systems is still open. Moreover, investigating the more sophisticated energy harvesting model, e.g., the nonlinear energy harvesting model, and the distributed user scheduling for these new models with imperfect CSI has more practical value and will be of interest for future research. 
Author Contributions: Conceptualization, Y.R. and M.M.; methodology, Y.R. and M.M.; investigation, Y.R. and X.Z.; writing-original draft preparation, Y.R.; writing-review and editing, X.Z.; supervision, X.Z. All authors have read and agreed to the published version of the manuscript.

Funding: This work was supported in part by the National Natural Science Foundation of China under Grants 61801382, 62101442 and 62071377, in part by the Natural Science Foundation of Shaanxi Province under Grants 2020JQ-849 and 2021JQ-705, and in part by the Young Talent fund of University Association for Science and Technology in Shaanxi, China under Grant 20210115.

Conflicts of Interest: The authors declare no conflict of interest.

\section{References}

1. Tang, M.; Gao, L.; Huang, J. Communication, Computation, and Caching Resource Sharing for the Internet of Things. IEEE Commun. Mag. 2020, 58, 75-80. [CrossRef]

2. Dastjerdi, A.V.; Buyya, R. Fog Computing: Helping the Internet of Things Realize Its Potential. Computer 2016, 49, 112-116. [CrossRef]

3. Cadambe, V.; Jafar, S. Interference alignment and degrees of freedom of the K user interference channel. IEEE Trans. Inf. Theory 2008, 54, 3425-3441. [CrossRef]

4. Zhao, N.; Yu, F.N.; Jin, M.; Yan, Q.; Leung, V.C.M. Interference Alignment and Its Applications: A Survey, Research Issues, and Challenges. IEEE Commun. Surv. Tut. 2016, 18, 1779-1803. [CrossRef]

5. Gou, T.; Jafar, S.A. Degrees of Freedom of the K-user M $\times$ N MIMO interference channel. IEEE Trans. Inf. Theory 2010, 56, 6040-6057. [CrossRef]

6. Suh, C.; Tse, D. Interference Alignment for Cellular Networks. In Proceedings of the 2008 46th Annual Allerton Conference on Communication, Control, and Computing, Monticello, IL, USA, 23-26 September 2008; pp. 1037-1044.

7. Ahn, S.K.; Chae, S.H. Blind Integer-Forcing Interference Alignment for Downlink Cellular Networks. IEEE Commun. Lett. 2019, 23, 306-309. [CrossRef]

8. Lv, W.; Wang, R.; Wu, J.; Xu, J.; Li, P.; Dou, J. Degrees of Freedom of the Circular Multirelay MIMO Interference Channel in IoT Networks. IEEE Internet Things J. 2018, 5, 1957-1966. [CrossRef]

9. Wan, X.; Zhu, X.; Jiang, Y.; Liu, Y.; Zhao, J. An Interference Alignment and ICA-Based Semiblind Dual-User Downlink NOMA System for High-Reliability Low-Latency IoT. IEEE Internet Things J. 2020, 7, 10837-10851. [CrossRef]

10. Castanheira, D.; Silva, A.; Gameiro, A. Set Optimization for Efficient Interference Alignment in Heterogeneous Networks. IEEE Trans. Wirel. Commun. 2014, 13, 5648-5660. [CrossRef]

11. Sheng, J.; Tang, Z.; Wu, C.; AI, B.; Wang, Y. Game Theory-Based Multi-Objective Optimization Interference Alignment Algorithm for HSR 5G Heterogeneous Ultra-Dense Network. IEEE Trans. Veh. Tech. 2020, 69, 13371-13382. [CrossRef]

12. Xu, F.; Tao, M.; Zheng, T. Cache-Aided Interference Management in Partially Connected Linear Networks. IEEE Trans. Commun. 2020, 68, 301-316. [CrossRef]

13. Zhang, H.; Yang, K.; Zhang, S.; Dobre, O.A. Subchannel Allocation Based on Clustered Interference Alignment for Differentiated Data Streams in Dense Small Cell Networks. IEEE Trans. Veh. Tech. 2020, 69, 14049-14054. [CrossRef]

14. Yang, H.J.; Shin, W.Y.; Jung, B.C.; Paulraj, A. Opportunistic Interference Alignment for MIMO Interfering Multiple-Access Channels. IEEE Trans. Wirel. Commun. 2013, 12, 2180-2192. [CrossRef]

15. Jung, B.C.; Kim, S.M.; Yang, H.J.; Shin, W.Y. On the Joint Design of Beamforming and User Scheduling in Multi-Cell MIMO Uplink Networks. In Proceedings of the 2014 IEEE 25th Annual International Symposium on Personal, Indoor, and Mobile Radio Communication (PIMRC), Washington, DC, USA, 2-5 September 2014; pp. 428-432.

16. Cha, H.S.; Jeon, S.W.; Kim, D.K. Blind Interference Alignment for the K-User MISO BC Under Limited Symbol Extension. IEEE Trans. Signal Process. 2018, 66, 2861-2875. [CrossRef]

17. Arti, M.K. A Novel Downlink Interference Alignment Method for Multi-User MIMO System With No CSIT: A Space-Time Coding Approach. IEEE Trans. Veh. Tech. 2020, 69, 10937-10948. [CrossRef]

18. Johnny, M.; Vahid, A. Exploiting Coherence Time Variations for Opportunistic Blind Interference Alignment. IEEE Trans. Commun. 2020, 68, 6054-6069. [CrossRef]

19. Ayach, O.E.; Heath, R.W. Interference Alignment with Analog Channel State Feedback. IEEE Trans. Wirel. Commun. 2012, 11, 626-636. [CrossRef]

20. Chen, X.; Yuen, C. Performance Analysis and Optimization for Interference Alignment over MIMO Interference Channels with Limited Feedback. IEEE Trans. Signal Process. 2014, 62, 1785-1795. [CrossRef]

21. Castanheira, D.; Silva, A.; Gameiro, A. Retrospective Interference Alignment for the K-User $M \times N$ MIMO Interference Channel. IEEE Trans. Wirel. Commun. 2016, 15, 8368-8379. [CrossRef]

22. Shahjehan, W.; Shah, S.W.; Lloret, J.; Bosch, I. Joint Interference and Phase Alignment among Data Streams in Multicell MIMO Broadcasting. Appl. Sci. 2018, 8, 1237. [CrossRef]

23. Lee, J.; Choi, W. On the Achievable DoF and User Scaling Law of Opportunistic Interference Alignment in 3-Transmitter MIMO Interference Channels. IEEE Trans. Wirel. Commun. 2013, 12, 2743-2753. [CrossRef] 
24. Leithon, J.; Yuen, C.; Suraweera, H.A.; Gao, H. A New Opportunistic Interference Alignment Scheme and Performance Comparison of MIMO Interference Alignment with Limited Feedback. In Proceedings of the 2012 IEEE Globecom Workshops, Anaheim, CA, USA, 3-7 December 2012; pp. 1123-1127.

25. Yang, H.J.; Shin, W.Y.; Jung, B.C.; Suh, C.; Paulraj, A. Opportunistic Downlink Interference Alignment for Multi-Cell MIMO Networks. IEEE Trans. Wirel. Commun. 2017, 16, 1533-1548. [CrossRef]

26. Suo, L.; Li, J.; Li, H.; Zhang, S.; Davidson, T.N. Achievable Sum Rate and Degrees of Freedom of Opportunistic Interference Alignment in MIMO Interfering Broadcast Channels. IEEE Trans. Commun. 2019, 67, 4062-4073. [CrossRef]

27. Jung, B.C.; Shin, W.Y. Opportunistic Interference Alignment for Interference-Limited Cellular TDD Uplink. IEEE Commun. Lett. 2011, 15, 148-150. [CrossRef]

28. Jung, B.C.; Park, D.; Shin, W.Y. Opportunistic Interference Mitigation Achieves Optimal Degrees-of-Freedom in Wireless Multi-Cell Uplink Networks. IEEE Trans. Commun. 2012, 60, 1935-1944. [CrossRef]

29. He, Y.; Zhang, Z.; Yu, F.R.; Zhao, N.; Yin, H.; Leung, V.C.M.; Zhang, Y. Deep-Reinforcement-Learning-Based Optimization for Cache-Enabled Opportunistic Interference Alignment Wireless Networks. IEEE Trans. Veh. Tech. 2017, 66, 10433-10445. [CrossRef]

30. Benaya, A.M.; Elsabrouty, M.; Muta, O. Queue-Aware Opportunistic Interference Alignment in Downlink MU-MIMO Cellular Systems. IEEE Access 2018, 6, 50860-50874. [CrossRef]

31. Ren, Y.; Lv, T.; Gao, H.; Li, Y. Secure Wireless Information and Power Transfer in Heterogeneous Networks. IEEE Access 2017, 5, 4967-4979. [CrossRef]

32. Zhang, X.; Lv, T.; Ren, Y.; Lin, Z. Joint Content Push and Transmission in NOMA With SWIPT Caching Helper. IEEE Commun. Lett. 2020, 24, 922-925. [CrossRef]

33. Zhao, N.; Yu, F.R.; Leung, V.C.M. Simultaneous Wireless Information and Power Transfer in Interference Alignment Networks. IEEE Wirel. Commun. 2015, 22, 88-95. [CrossRef]

34. Zhao, N.; Yu, F.R.; Leung, V.C.M. Wireless Energy Harvesting in Interference Alignment Networks. IEEE Commun. Mag. 2015, 53, 72-78. [CrossRef]

35. Koo, B.; Park, D. Interference Alignment and Wireless Energy Transfer via Antenna Selection. IEEE Commun. Lett. 2014, 18, 548-551. [CrossRef]

36. Zhao, N.; Yu, F.R.; Leung, V.C.M. Wireless Power Transfer Based on Angle Switching in Interference Alignment Wireless Networks. In Proceedings of the 2015 IEEE International Conference on Communications (ICC), London, UK, 8-12 June 2015; pp. 3776-3781.

37. Xie, Z.; Chen, Y.; Gao, Y. Joint Iterative Interference Alignment and Energy Harvesting for Multi-User Networks. IEEE Wirel. Commun. Lett. 2015, 4, 597-600. [CrossRef]

38. Horn, R.; Johnson, C. Matrix Analysis; Cambridge University Press: New York, NY, USA, 1990.

39. Gao, H.; Leithon, J.; Yuen, C.; Suraweera, H.A. New Uplink Opportunistic Interference Alignment: An Active Alignment Approach. In Proceedings of the 2013 IEEE Wireless Communications and Networking Conference (WCNC), Shanghai, China, 7-10 April 2013; pp. 3066-3071.

40. Hur, S.H.; Jung, B.C.; Rao, B.D. Sum rate enhancement by maximizing SGINR in an opportunistic interference alignment scheme. In Proceedings of the 2011 Conference Record of the Forty Fifth Asilomar Conference on Signals, Systems and Computers (ASILOMAR), Pacific Grove, CA, USA, 6-9 November 2011; pp. 354-358.

41. Lv, T.; Gao, H.; Cao, R.; Zhou, J. Co-Ordinated Secure Beamforming in K-User Interference Channel With Multiple Eavesdroppers. IEEE Wirel. Commun. Lett. 2016, 5, 212-215. [CrossRef] 